\title{
The Inequality of Work Derived from Clausius Inequality and Carnot Theorem
}

\author{
Chengshu Jin ${ }^{*}$ \\ Department of Food and Pharmaceutical Engineering, Suihua University, Suihua, Heilongjiang Province 152061, China \\ ${ }^{*}$ Corresponding author
}

\begin{abstract}
According to the Clausius inequality, it can be proven that the quotient of work-temperature in the real process is no less than one in the reversible process. On the basis of the Carnot theorem, it can be proven that the work in the real process is no less than one in the reversible process, or the heat in the reversible process is no less than one in the real process. The real process includes the reversible and irreversible process. The above-mentioned results haven't any relation with paths. The inequality of quotient of work-temperature could be applied to other works. The $\mathrm{He}(\mathrm{II})$ motion in superfluid obeys the inequality of quotient of work-temperature.
\end{abstract}

Keywords-the first law; Clausius inequality; Carnot theorem; work; heat

\section{INTRODUCTION}

Mathematical formulation of the second law of thermodynamics is the Clausius inequality [1-9], which is derived from the Carnot theorem.

The first law of thermodynamics is given by

$$
\mathrm{dU}=\delta \mathrm{Q}_{\mathrm{r}}+\delta \mathrm{W}_{\mathrm{r}}
$$

where, $\mathrm{U}$ is the internal energy. $\mathrm{Q}_{\mathrm{r}}$ and $\mathrm{W}_{\mathrm{r}}$ are the heat and work in the reversible process respectively. $\mathrm{Q}_{\text {real }}$ and $\mathrm{W}_{\text {real }}$ are the heat and work in the real process respectively. The real process includes the reversible and irreversible process. The heat and work are positive if energy is gained by the system as heat and work respectively, and negative if energy is lost from the system as heat or work respectively. Because $\mathrm{dU}=\mathrm{dU}_{\text {real }}$, we have

$$
\mathrm{dU}_{\text {real }}=\delta \mathrm{Q}_{\text {real }}+\delta \mathrm{W}_{\text {real }}
$$

The heat and work are the path function. $U$ is the state function. " $d "$ is differential symbol. " $\delta$ (delta)" is inexact differential symbol.

The criteria for the real or spontaneous process is the Clausius inequality, it can be written as

$$
\int_{j}^{i \delta Q_{r}(A)} \frac{i \delta Q_{\text {real }}(B)}{T}-\int_{j} \frac{0}{T_{i}}
$$

where, the inequality in Eq. (3) is for the irreversible, and the equality in Eq. (3) is for the reversible process. $\int_{j}^{i \delta Q_{r}(A)} \frac{1}{T}$ is the quotient of heat-temperature in the reversible process for "A" path, and defined as the entropy change $\Delta S . \int_{j}^{i \delta Q_{\text {real }}(B)} \frac{T_{i}}{T_{i}}$ is the quotient of heat-temperature in the real process for " $\mathrm{B}$ " path. The reversible process is from $\mathrm{j}$ state to $\mathrm{i}$ state in " $\mathrm{A}$ " path, $\mathrm{j}$ is the initial state. The real process is from $\mathrm{j}$ state to $\mathrm{i}$ state in " $\mathrm{B}$ " path, $\mathrm{i}$ is the final state. $\mathrm{T}$ is the temperature for the system or reservoir, $T_{j}$ is the initial state temperature, $T_{i}$ is the final state temperature.

\section{THE INEQUALITY OF WORK AND QUOTIENT OF WORK- TEMPERATURE}

In the reversible process of Carnot cycle, the efficiency of heat engine is

$$
\eta=\frac{-\mathrm{W}_{\mathrm{r}}(\mathrm{A})}{\mathrm{Q}_{1}(\mathrm{~A})}
$$

where, $\mathrm{W}_{\mathrm{r}}(\mathrm{A})$ indicates the total work in the reversible process of Carnot cycle (it is negative). $Q_{1}(A)$ is the heat of absorption in the isothermal expansion process of reversible Carnot cycle (it is positive)

In the irreversible process of Carnot cycle, the efficiency of heat engine is

$$
\eta^{\prime}=\frac{-\mathrm{W}_{\mathrm{ir}}(\mathrm{B})}{\mathrm{Q}_{2}(\mathrm{~B})}
$$

where, $\mathrm{W}_{\mathrm{ir}}(\mathrm{B})$ indicates the total work in the irreversible process of Carnot cycle (it is negative). $\mathrm{Q}_{2}(\mathrm{~B})$ is the heat of absorption in the isothermal expansion process of irreversible Carnot cycle (it is positive).

According to the Carnot theorem, the efficiency of heat engine of irreversible processes is less than one of reversible processes, the result can be expressed as follows 


$$
\frac{-\mathrm{W}_{\mathrm{r}}(\mathrm{A})}{\mathrm{Q}_{1}(\mathrm{~A})}>\frac{-\mathrm{W}_{\mathrm{ir}}(\mathrm{B})}{\mathrm{Q}_{2}(\mathrm{~B})} \quad \frac{-\mathrm{W}_{\mathrm{r}}(\mathrm{A})}{\text { or }}>\frac{\mathrm{Q}_{1}(\mathrm{~A})}{-\mathrm{W}_{\mathrm{ir}}(\mathrm{B})}
$$

It is impossible that $\mathrm{Q}_{1}(\mathrm{~A})<\mathrm{Q}_{2}(\mathrm{~B})$ in the isothermal expansion process, that is,

$$
\int_{j}^{i} \frac{\delta Q_{1}(A)}{T}-\int_{j}^{i} \frac{\delta Q_{2}(B)}{T_{i}}=\int_{j}^{i} \frac{\delta Q_{1}(A)}{T_{i}}-\int_{j}^{i} \frac{\delta Q_{2}(B)}{T_{i}}<0
$$

which violates the Clausius inequality. Therefore, the result is $\mathrm{Q}_{1}(\mathrm{~A})>\mathrm{Q}_{2}(\mathrm{~B})$ and $\mathrm{W}_{1}(\mathrm{~A})<\mathrm{W}_{2}(\mathrm{~B})$. Where, $\mathrm{W}_{1}(\mathrm{~A})$ and $\mathrm{W}_{2}(\mathrm{~B})$ are the work done by the isothermal expansion process of reversible and irreversible Carnot cycle in high temperature respectively. On the basis of the Carnot theorem, $\frac{-\mathrm{W}_{\mathrm{r}}(\mathrm{A})}{-\mathrm{W}_{\mathrm{ir}}(\mathrm{B})}>\frac{\mathrm{Q}_{1}(\mathrm{~A})}{\mathrm{Q}_{2}(\mathrm{~B})}>1$, namely that, $\mathrm{W}_{\mathrm{r}}(\mathrm{A})<\mathrm{W}_{\mathrm{ir}}(\mathrm{B})$.

Therefore, we can obtain

$$
\mathrm{W}_{\mathrm{r}}(\mathrm{A}) \leq \mathrm{W}_{\text {real }}(\mathrm{B})
$$

On the basis of the first law, the following formulation can be given by

$$
\mathrm{Q}_{\mathrm{r}}(\mathrm{A}) \geq \mathrm{Q}_{\text {real }}(\mathrm{B})
$$

where, the inequality in the equations $(7,8)$ are for the irreversible process, and the equality in the equations $(7,8)$ are for the reversible process. $\mathrm{Q}_{\mathrm{r}}(\mathrm{A})$ and $\mathrm{Q}_{\text {real }}(\mathrm{B})$ express the total heat in the reversible and real process of the Carnot cycle respectively.

Assuming that the real process and its reversible process constitute a cycle, the equations $(7,8)$ can be extended and applied to any cycle or process.

$$
\text { If } \int_{j}^{i} \frac{\delta W_{r}(A)}{T}-\int_{j}^{i} \frac{\delta W_{\text {real }}(B)}{T_{i}}>0 \text { were correct, the result }
$$

would be $\mathrm{W}_{\mathrm{r}}(\mathrm{A})>\mathrm{W}_{\text {real }}(\mathrm{B})$ in the isothermal process, which disobeys Eq. (7) or the Clausius inequality and the Carnot theorem. Therefore, this hypothesis is a error.

So that, we can obtain

$$
\int_{j}^{i} \frac{\delta W_{r}(A)}{T}-\int_{j}^{i} \frac{\delta W_{\text {real }}(B)}{T_{i}} \leq 0
$$

where, the inequality in Eq. (9) is for the irreversible process, and the equality in Eq. (9) is for the reversible process. Eq. (9) obeys Eq. (7) or the Clausius inequality and the Carnot theorem. $\int_{\mathrm{j}}^{\mathrm{i} \delta \mathrm{W}_{\mathrm{r}}(\mathrm{A})} \mathrm{T}$ is the quotient of work-temperature in the reversible process for " $\mathrm{A}$ " path. $\int_{\mathrm{j}}^{\mathrm{i}} \frac{\delta \mathrm{W}_{\text {real }}(\mathrm{B})}{\mathrm{T}_{\mathrm{i}}}$ is the quotient of work-temperature in the real process for "B" path.

\section{CONCLUSIONS}

It is impossible that $\mathrm{Q}_{\mathrm{r}}<\Delta \mathrm{H}$ for the chemical reactions in the isothermal and isobaric condition, because the condition of chemical reactions occurred is $\Delta \mathrm{H}-\mathrm{T} \Delta \mathrm{S}=\Delta \mathrm{G} \leq 0$. Where, $\mathrm{H}$ is the enthalpy, $\mathrm{G}$ is the free energy. So that, $\mathrm{Q}_{\mathrm{r}} \geq \Delta \mathrm{H}\left(\mathrm{Q}_{\mathrm{r}}=\mathrm{T} \Delta \mathrm{S}\right.$, $\left.\mathrm{Q}_{\text {real }}=\Delta \mathrm{H}\right) . \quad \mathrm{W}_{\mathrm{r}} \leq \mathrm{W}_{\text {real }}$, since $\quad \Delta \mathrm{U}=\mathrm{Q}_{\mathrm{r}}+\mathrm{W}_{\mathrm{r}}=\mathrm{Q}_{\text {real }}+\mathrm{W}_{\text {real. }} . \quad$ The equations $(7,8)$ are obeyed.

The ideal gas expands to vacuum container in the isochoric and adiabatic process, $\Delta \mathrm{U}=\mathrm{W}_{\text {real }}=0$ and $\mathrm{Q}_{\text {real }}=0$ in the real process. The reversible process is the isothermal expansion process, the results are $\mathrm{Q}_{\mathrm{r}}>0$ and $\mathrm{W}_{\mathrm{r}}<0$. Therefore, the equations $(3,7-9)$ are all obeyed.

The work is relation with negative value of the kinetic and potential energy [8-10] in the reversible process. If the potential energy doesn't exist, we have

$$
\mathrm{W}_{\mathrm{r}}=-\mathrm{E}_{\mathrm{kin}}
$$

Namely that

$$
\int_{\mathrm{j}}^{\mathrm{i}} \frac{\mathrm{dE}_{\text {kin }}}{\mathrm{T}} \geq \int_{\mathrm{j}}^{\mathrm{idE_{ \text {kin } }}} \frac{\mathrm{T}_{\mathrm{i}}}{\mathrm{T}^{\prime}}
$$

where, $E_{\text {kin }}$ and $E_{\text {kin }}{ }^{\prime}$ indicate the kinetic energy in the reversible and real process respectively. The inequality in Eq. (11) is for the irreversible process, and the equality in Eq. (11) is for the reversible process. Here, $E_{k i n}=\frac{{m v_{i}}^{2}}{2}-\frac{m_{j}{ }^{2}}{2}$, $\mathrm{E}_{\mathrm{kin}}{ }^{\prime}=\frac{\mathrm{m}\left(\mathrm{v}_{\mathrm{i}}^{\prime}\right)^{2}}{2}-\frac{\mathrm{m}\left(\mathrm{v}_{\mathrm{j}}^{\prime}\right)^{2}}{2}, \mathrm{v}$ express the velocity.

If $\mathrm{Q}_{\text {real }}=\mathrm{Q}_{\mathrm{r}}$ (all of work can be neglected), the criteria for the real or spontaneous process is Eq. (3). For example, the heat conduction process, the thermal radiation process, or the temperature risen or fallen process for the isochoric gases, solids and liquids.

If $\mathrm{W}_{\text {real }}=\mathrm{W}_{\mathrm{r}}$ (the resistance such as friction or internal resistance or air resistance can be neglected), the criteria for the real or spontaneous process is Eq. (9). For instance, the $\mathrm{He}(\mathrm{II})$ motion in superfluid, or the electron motion in superconductor.

$\mathrm{Q}_{\text {resist }}$ and $\mathrm{W}_{\text {resist }}$ are the heat and work yielded by resistance or path in the real process respectively. According to the equations $(7,8)$, we gain $-Q_{\text {resist }} \geq 0, W_{\text {resist }} \geq 0$. From the equations $(1,2)$, we have $\mathrm{Q}_{\text {resist }}+\mathrm{W}_{\text {resist }}=0$. Therefore 


$$
\mathrm{Q}_{\text {real }}=\mathrm{Q}_{\mathrm{r}}+\mathrm{Q}_{\text {resist }} \text { or } \mathrm{W}_{\text {real }}=\mathrm{W}_{\mathrm{r}}+\mathrm{W}_{\text {resist }} \text {. }
$$

It is consistent with the actual fact, $\mathrm{Q}_{\text {resist }}$ is negative and releases the heat in the irreversible process [10].

The van der Waals equation of state is an approximate estimate for real gases. The ideal gas equation of state only is corret for ideal gas. So that, $\int_{\mathrm{j}}^{\mathrm{i} \delta \mathrm{W}_{\mathrm{r}}} \mathrm{T}$ and $\int_{\mathrm{j}}^{\mathrm{idU}} \frac{\mathrm{r}}{\mathrm{T}}$ are all the state function. When the work exists, Eq. (9) is equivalent with Eq. (3) or the Clausius inequality.

\section{REFERENCES}

[1] C. Borgnakke, R. E. Sonntag, Fundamentals of thermodynamics, eighth ed., Singapore: John Wiley \& Sons Singapore Pte. Ltd, 2014.

[2] A. Burrows, J. Holman, A. Parsons, G. Pilling, G. Price, Chemistry 3: Introducing Inorganic, Organic and Physical Chemistry, second ed., Oxford: Oxford Univerity press, 2013.

[3] P. Atkins, J. de Paula, Atkins' physical chemistry, tenth ed., Oxford: Oxford University Press, 2014.

[4] C. Jin, The rules of clusters and the second law of thermodynamics (in Chinese), Haerbin: Heilongjiang Science \& Technology Press, 2016.

[5] S. I. Sandler, L. V. Woodcock, "Historical observations on laws of thermodynamics,” J. Chem. Eng. Data, Vol.55, pp. 4485-4490, 2010.

[6] S. Lee, K. Lee, J. Lee, "An alternative presentation of the second law of thermodynamics," J. Chem. Educ., Vol.92, pp. 771-773, 2015.

[7] R. Nieto, C. González, Á. Jiménez, I. López, J. Rodríguez, “A missing deduction of the Clausius equality and inequality," J. Chem. Educ., Vol.88, pp. 597-601, 2011.

[8] E. A. Gislason, N. C. Craig, "Criteria for spontaneous processes derived from the global point of view," J. Chem. Educ., Vol.90, pp. 584-590, 2013.

[9] R. de Abreu, V. Guerra, "Introducing thermodynamics through energy and entropy,” Am. J. Phys., Vol.80, pp. 627-637, 2012.

[10] H. DeVoe, "A comparison of local and global formulations of thermodynamics," J. Chem. Educ., Vol.90, pp. 591-597, 2013. 